\title{
Baseline Drift Removal and De-Noising of the ECG Signal using Wavelet Transform
}

\author{
Iffat Ara \\ Lecturer \\ Dept. of ICE \\ Pabna University of Science \\ and Technology, Bangladesh
}

\author{
Md. Najmul Hossain \\ Dept. of ETE \\ Pabna University of Science \\ and Technology, Bangladesh
}

\author{
S. M. Yahea Mahbub \\ Dept. of ETE \\ Pabna University of Science \\ and Technology, Bangladesh
}

\begin{abstract}
ECG signal plays an important role in the primary diagnosis and analysis of heart diseases. When an Electrocardiogram is recorded many kinds of noise are recorded. The aim of this paper is to use discrete wavelet transform (DWT) for denoising the ECG signal. Text formatted ECG signals of ten second duration are taken from the MIT-BIH arrhythmia database. ECG signal of Modified lead II (MLII) are chosen for processing. For wavelet transform, daubechies wavelets were used because the scaling functions of this wavelet filter are similar to the shape of the ECG. From the decomposition of the ECG signal it was seen that the low frequency component cause the baseline shift, theses component were deducted to get a signal without baseline drift. Also the high frequency components of the signal were removed for getting denoised signal. A program has been developed with MATLAB software for this work.
\end{abstract}

\section{General Terms}

Biomedical Signal Processing

\section{Keywords}

ECG, Wavelet transform, P_QRS-T waves, Baseline drift, Denoising.

\section{INTRODUCTION}

The ECG is nothing but the recording of the heart's electrical activity that is generated by depolarization and repolarization of the atria and ventricales [01]. ECG is an important tool for the primary diagnosis of heart disease; it shows the electrophysiology of the heart and the ischemic changes that may occur like the myocardial infection, conduction defects and arrhythmia [02]. One cardiac cycle in an ECG signal consists of the P-QRS-T waves. When an ECG signal is recorded many kinds of noise are recorded such as: Base line drift, power line interference, motion artifact. These noise can make clinical diagnosis very difficult. DWT has been described as a good tool for the reduction of ECG signal baseline drift. DWT decomposes a signal at different scale or resolution.

\section{MATERIAL AND METHOD}

The wavelet transform is a convolution of the wavelet function $\psi(\mathrm{t})$ with the signal $\mathrm{x}(\mathrm{t})$. Orthonormal dyadic discrete wavelets are associated with scaling function $\varphi(t)$. The scaling function can be convolved with the signal to produce approximation coefficients $\mathrm{S}$. The discrete wavelet transforms (DWT) can be written as:

$$
T_{m, n}=\int_{-\infty}^{\infty} x(t) \psi_{m n}(t) d t
$$

Where $T_{m_{n}}$ is known as the wavelet (or detail) coefficient at scale and location indices $(m, n)$.

By choosing an orthonormal wavelet basis, $\psi \mathrm{m}, \mathrm{n}(\mathrm{t})$, and we can reconstruct the original. The approximation coefficients of the signal at the scale $\mathrm{m}$ and location $\mathrm{n}$ can be represented by:

$S_{m, n}=\int_{-\infty}^{\infty} x(t) \varphi_{m n}(t) d t$

The discrete input signal $S_{0_{n} n}$ is of finite length $\mathrm{N}$, which is an integer power of $2: N=2^{\mathrm{m}}$. Thus the range of scales that can be investigated is $0<\mathrm{m}<\mathrm{M}$. A discrete approximation of the signal can be shown as

$$
x_{0}(t)=x_{M}(t)+\sum_{m=1}^{M} d_{m}(t)
$$

Where the mean signal approximation at scale $\mathrm{M}$ is

$x_{M}(t)=S_{M_{n} n} \varphi_{M_{n}}(t)$

The detail signal approximation corresponding to scale index $\mathrm{m}$ is defined for a finite length signal as

$$
d_{m}(t)=\sum_{n=0}^{2^{M-m}-1} T_{m, n} \psi_{m n}(t)
$$

The signal approximation at a specific scale is a combination of the approximation and detail at the lower scale

$$
x_{m}(t)=x_{m-1}(t)-d_{m}(t) .
$$

If scale $m=3$ was chosen, it can be shown that the signal approximation is given by

$x_{a}(t)=x_{0}(t)-d_{1}(t)-d_{2}(t)-d_{a}(t)$

Corresponding to the successive stripping of high frequency information (contained within the $\mathrm{dm}(\mathrm{t})$ ) from the original signal at each step [03]. This is referred to as multi-resolution analysis of a signal using wavelet transform, and is the basic of our procedure.

\subsection{Prototype Wavelet Used}

The large number of known wavelet families and functions provides a rich space in which to search for a wavelet which will very efficiently represent a signal of interest in a large variety of applications. Wavelet families include Biorthogonal, Coiflet, Haar, Symmlet, Daubechies wavelets [04], etc. There is no absolute way to choose a certain wavelet. The choice of the wavelet function depends on the application. The Haar wavelet algorithm has the advantage of being simple to compute and easy to understand. The Daubechies algorithm is conceptually more complex and has a slightly higher computational overhead. But, the Daubechies 
algorithm picks up detail that is missed by the Haar wavelet algorithm. Even if a signal is not well represented by one member of the $\mathrm{Db}$ family, it may still be efficiently represented by another. Selecting a wavelet function which closely matches the signal to be processed is of utmost importance in wavelet applications [05]. Daubechies wavelet families are similar in shape to QRS complex and their energy spectrums are concentrated around low frequencies.

\section{ECG PREPROCESSING}

When an Electrocardiogram is recorded many kinds of noises are also be recorded due to very low and high frequencies [06] , which causes an ECG to have baseline drift and noise in the signal and is very difficult to clinically diagnose. For proper diagnosis of ECG it is necessary to remove noise from the signal. A process of removing the baseline drift of a signal $[07,06]$ is called as de-trending and a process of removing the noise [06] of a signal is called as de-noising. Both of these processes come under the preprocessing of an ECG signal. Once the signal is preprocessed then it can be used for further processing.

Figure: 1 shows the details of the ECG signal. These signals are decomposed in eight level using db6 wavelets. The original signal is shown at the top and below the signal the details for eight wavelets scales is shown in figure:1. Adding together all these details plus the remaining signal approximation (which is the signal mean) returns the original signal. Four points are noticeable from the plots. First, most of the details of the signal are contained at scale 25 . The second point to notice is that the high frequency burst of noise is captured at the smallest scales, namely 22 and 21 . The third is the appearance of Daubechies D6 scaling functions at higher scales. The last one is the similarity of D6 scaling function to QRS complex, as it can be seen by the details of the signal at scale 25. Because of the latter property the D6 wavelet is implemented here.

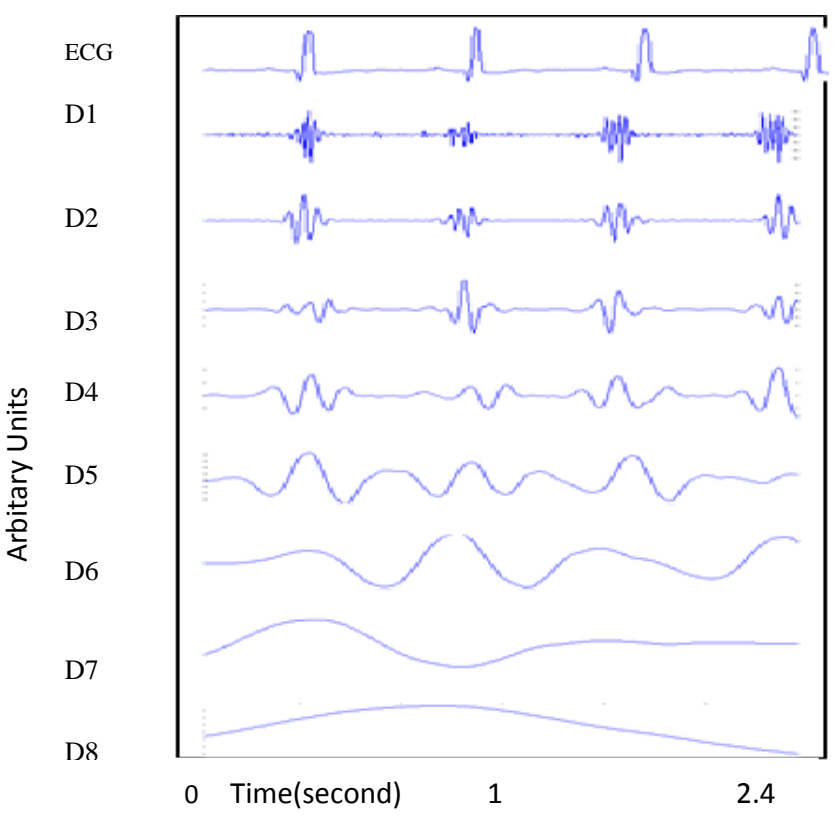

Figure1: Multiresolution decomposition of ECG signal using db6.

.If the frequency distribution of details is drawn it might be found better understanding of wavelet transform. A normal ECG signal along with its frequency distribution is shown in Figure:2. The signal is sampled at 360 sample/second, so the range of real frequency component of the signal is between 0 to $180 \mathrm{~Hz}$. A normal signal is used here to omit the interference of abnormal beats in frequency distribution. The frequency response of the original signal shows its main concentration in the lower frequency range $2-40 \mathrm{~Hz}$. The signal had a large de component which was removed to enhance other frequency components of the signal. The frequency response of the wavelets details is also shown which are exaggerated to clearly show their distribution.

It can be seen that details at lower scales constitutes the higher frequencies of the signal. Although the frequency distribution of the details does not overlap each other at lower scales up to $2^{3}$, they obviously overlap at higher scales. While working with details of the signal, these overlaps between discrete distributions should be taken into consideration.

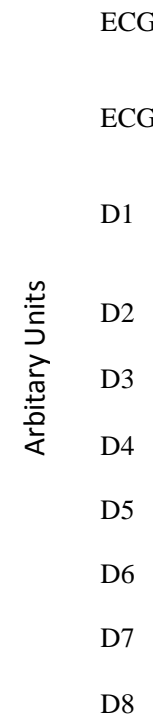

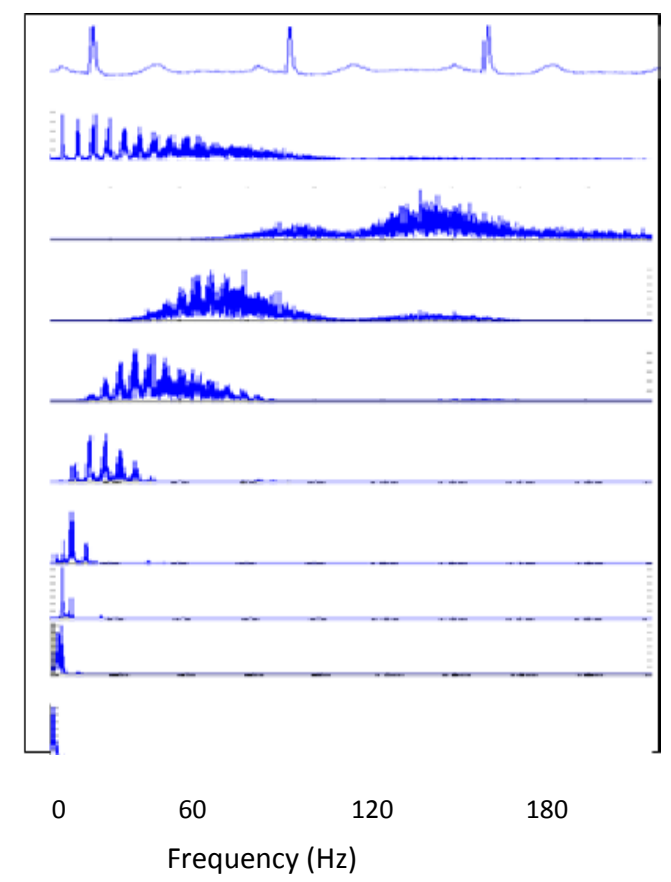

Figure 2: A normal (16265 mitdb) ECG signal and its frequency distribution.

\subsection{Baseline drift removal and denoising of the ECG signal}

A process of removing the baseline drift of a signal is called as de-trending and a process of removing the noise of a signal is called as de-noising. Both of these processes come under the preprocessing of an ECG signal. Once the signal is preprocessed then it can be used for further processing. The developed algorithm is implemented using MATLAB 7.5b. From the entire MIT-BIH database signal which we used for analysis, removed the baseline drift and denoised the signal. But the figure of only one denoising and baseline drift removing signal is given here. The original ECG signals of length 800 samples are shown in shown in Figure-3.

Since the low frequency components cause the signal for baseline shifting, these components must be deducted to have a signal without baseline drift. In this study, the low frequency components of a decomposed signal are A8 and D8. Therefore, to remove the baseline Drift these components should be removed from the original ECG signal. i.e. De-trended Signal $(\mathrm{DS})=$ Original Signal $-(\mathrm{A} 8+\mathrm{D} 8)$. Thus 
the problem of baseline shifting is solved. The de-trended ECG signal of length 800 samples is shown Figure-4.

Though the low frequency components are removed from the original signal, still it may have noise due to high frequency components. In this study the identified high frequency components are D1, D2. These components must be filtered by applying a threshold. Then the threshold components are removed from the de-trended signal. The de-noised ECG signal of length 800 samples is shown in Figure-

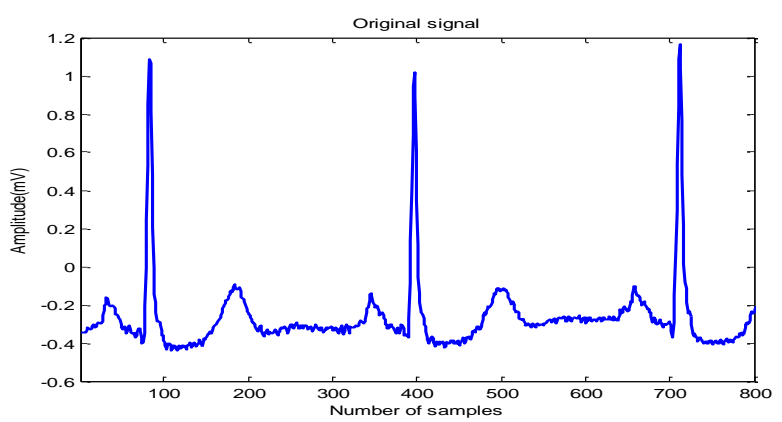

Figure-3: Original ECG signal of the record 101, MLII
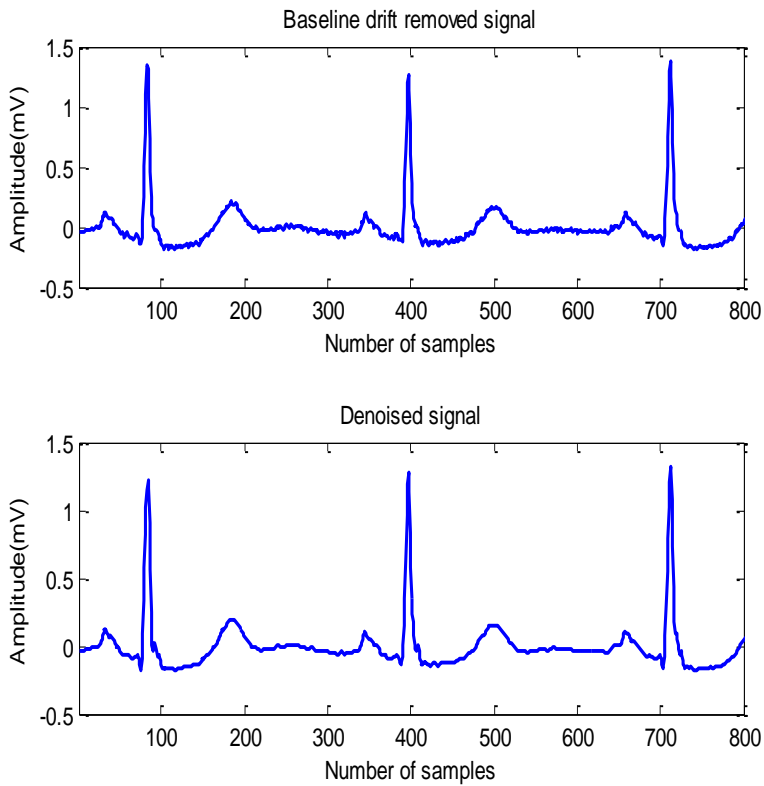

Figure-4: Baseline drift removal and de-noised signal of record 101, MLII

\section{RESULT AND DISCUSSION}

The wavelet analysis tool as well as the graphical result for different normal and abnormal ECG signal is presented. The wavelet analysis of ECG signal is performed using MATLAB software. MATLAB is a high performance; interactive system which allows to solve many technical computing problem. MATLAB 7.5 versions have been used to write computer program designed for analyzing.

\section{CONCLUSION}

By decomposing of the signal it has been seen that most of the details of the signal are contained at lower scale which need less decomposition, so faster application of the wavelet. The decomposition levels depend on the length of the signal. The absence of very low and very high frequency concentration of the signal helped us to de-noise the signal from movement artifacts and external interfering noise easily.The database signal should be de-noised for detecting features properly. Two records(102 and 104) of the database were not used because they do not contain the signal of MLII lead.

\section{REFERENCES}

[1] L. Cromwell, F.J. Weibell, and E.A. Pfeiffer: Biomedical Instrumentation and Measurements, Prentice Hall of India, New Delhi 2005.

[2] Abdel-Rahman, Al-Qawasmi and Khaled Daqrouq: ECG signal enhancement Using Wavelet Transform, Published by WSEAS TRANSACTION on BIOLOGY and BIOMEDICINE; issue: 2; Volume: 7; pp: 62-72; April 2010.

[3] Paul S Addison, The illustrated wavelet transform handbook, (IOP Pub., 2002).

[4] Daubechies, I., Ten Lectures on Wavelets, SIAM, Philadelphia, 1992.

[5] A. Grap: An introduction to wavelets, IEEE Computing in science and Engineering, Vol: 2 (2); pp: 50-61, 1995.

[6] Vanisree K and Jyothi Singraraju: Automatic detection of ECG R-R interval using discrete wavelet transform , International Journal on Computer Science and Engineering (IJCSE);Vol:3;No:4;pp:1599-1605;April 4, 2011.

[7] K. Daqrouq: ECG baseline wandering reduction using wavelet transform, Asian journal of information Technology; Vol: 4(11); pp=989-995; 2005. 